\title{
Postkeynesianismus. Ein heterodoxer Ansatz auf der Suche nach einer Fundierung: Replik zum Kommentar von Johannes Becker
}

\author{
Arne Heise
}

Online publiziert: 28. Februar 2019

(C) List-Gesellschaft e.V. 2019

In den nachfolgenden Ausführungen möchte ich keine Replik im Sinne einer „,Gegenrede“, oder eines verbalen „Konters“ auf den Kommentar von Johannes Becker liefern, sondern die Chance ergreifen, jene Punkte weiter auszuführen, die entweder nicht hinreichend deutlich geworden sind oder nicht Gegenstand eines Artikels sein konnten, der sich in erster Linie mit der erläuternden Vorstellung einer Theorieschule befasste.

Beginnen aber muss ich mit einem offensichtlichen Missverständnis: Ich hoffte deutlich gemacht zu haben, dass ich gerade nicht - quasi zur Neutralität selbstverpflichtet - den bunten Strauß postkeynesianischer Theorievarianten vorstellen wollte, der den Leser oder ausgebildeten Laien doch einigermaßen ratlos dahingehend hätte zurücklassen müssen, worin denn nun die konstruktive Alternative zum DSGMParadigma besteht. Vielmehr habe ich mich auf die Seite eines Postkeynesianismus - der monetären Produktionsökonomik - geschlagen, um die identifizierten Grundbestandteile aller Postkeynesianismen in eine konkrete formale Struktur bringen zu können. Und die Opposition zu den Dynamisch-Stochastischen Allgemeinen Gleichgewichtsmodellen, die zusammen das dominante neoklassische Paradigma beschreiben, besteht weder in deren dynamischer Orientierung auf einen Gleichgewichtszustand, noch in der Forderung nach Mikrofundierung (wie das vorgestellte Modell ja zeigt), sondern in der Zurückweisung des für das DSGM-Paradigma konstituierenden Walras-Gesetzes. Und dies impliziert sowohl die Kritik an der un-

A commentary article to this article is available online at https://doi.org/10.1007/s41025-01900132-9.

\footnotetext{
A. Heise $(\bowtie)$

Universität Hamburg, Hamburg, Deutschland

E-Mail: Arne.Heise@uni-hamburg.de
} 
terstellten Stochastik der durch das Modell angenäherten Realität ${ }^{1}$, wie auch an der Allgemeinheit des Gleichgewichts - also der Notwendigkeit, dass sich partielle Ungleichgewichte immer exakt kompensieren. Hierin, in der Vorstellung, bei der zu untersuchenden ökonomischen Realität handele es sich um ein offenes, nicht-deterministisches System (statt eines geschlossenen, deterministischen Systems) auf der Grundlage nomineller Verpflichtungen (statt inter-temporaler Tauschvorgänge), zeigen sich die grundsätzlichen und unüberbrückbaren ontologischen Differenzen zwischen dem heterodoxen Postkeynesianismus und dem orthodoxen DSGM-Paradigma - weshalb Farmers Schöpfung eines postkeynesianischen DSGM ebenso unsinnig (weil in sich widersprüchlich) ist wie es nicht weiterhilft, diese paradigmatischen Differenzen dadurch negieren zu wollen, dass die Bedeutung von Namensgebungen oder Labels hinterfragt wird. Wir sollten uns in der Ökonomenzunft vielmehr wieder klarer darüber werden, dass es unterschiedliche prä-analytische Visionen wie es Schumpeter (1954, S. 41) nannte - geben kann, die die Grundlage potentiell inkompatibler und inkommensurabler Theoriegebäude sein können.

$\mathrm{Ob}$ eine derartige paradigmatische Alternative zum Mainstream-DSGM belastbar ist und mit Recht Ressourcen z. B. im Form von Professuren und Forschungsmitteln reklamieren kann, muss sich selbstverständlich darin zeigen, ob sie in der Lage ist, Phänomene und Probleme der ökonomischen Realität im Zweifel besser erklären zu können als der dominante Mainstream. Wer sich die Mühe macht - und Zitationsstudien zeigen, dass dies zumindest nicht in ausreichendem Maße für die Vertreter der Mainstream-Ökonomik gilt (vgl. Kapeller 2010a, 2010b) -, den heterodoxen Forschungsoutput in einschlägigen Fachjournals zu studieren, wird konstatieren müssen, dass sich die postkeynesianische Ökonomik genau an diesen Kriterien messen lassen will. Und zumindest für Postkeynesianer - vielleicht aber auch für unvoreingenommene ausgebildete Laien - lassen sich gleichermaßen z. B. die Existenz von dauerhafter Massenarbeitslosigkeit in allen hochentwickelten Volkswirtschaften, wie systematische Instabilitäten bis hin zum Systemkollaps (der ohne beherzte staatliche Interventionen jenseits der Mainstreamanalyse nach 2008 wohl eingetreten wäre) oder die starkköpfige Weigerung der Realität, bei Mindestlohneinführungen keine Massenarbeitslosigkeit zu produzieren, besser und überzeugender mit ihren Modellen erklären als mit den Myriaden von Mainstream-Theorien, die auf den Ausbruch kollektiver Faulheit, plötzlich und unerklärte Produktivitätsschocks oder monopsonistisches Verhalten in nicht-monopsonistischen Märkten setzen. Es sollen hier keine wissenschaftlichen Anstrengungen lächerlich gemacht werden, sondern lediglich die Anforderungen, die mit besonderem Nachdruck an die heterodoxen Theorien gestellt werden - als seien sie in einer Bringschuld gegenüber dem Mainstream! - an den Mainstream zurückgespiegelt werden.

\footnotetext{
1 Zugegebenermaßen bezieht sich die Stochastik-Eigenschaft zunächst auf das Modell, nicht die Realität. Und man kann durchaus - im Sinne der Komplexitätsreduktion - eine nicht-stochastische Realität durch ein stochastisches Modell zu erklären versuchen. Dies ist aber nur dann zulässig, wenn diese Art der Komplexitätsreduktion keinen Einfluss auf das Realitätsverständnis hat - was durch den Postkeynesianismus bestritten wird.
} 
Außerdem noch eine Anmerkung zur zunehmenden und weitreichenden Verdrängung heterodoxer Paradigmen aus dem wirtschaftswissenschaftlichen Markt (nicht nur) in Deutschland, die Becker nicht als Ausdruck der unfairen Marginalisierung in einem mit zahlreichen Formen des Marktversagens konfrontierten Marktgeschehen verstehen will (vgl. Heise 2016), sondern als Ergebnis einer offenbar „,misslungenen Reintegration“ in die Makroökonomik. Sollte mit „Reintegration“ das Verhaltensmuster gemeint sein, heterodoxe Ansätze dem Mainstream-Paradigma einzuverleiben - wie z.B. der Keynes'sche Ansatz der Allgemeinen Theorie zum „Standardkeynesianismus" synthetisiert wurde (vgl. z. B. Davidson 2007, S. $169 \mathrm{ff}$. .) -, dann ist genau diese Reintegration aus den oben genannten Gründen abzulehnen und die folgende Marginalisierung - also Nicht-Beachtung - ist dann eben nicht das Ergebnis eines Versagens des Postkeynesianismus nach den methodologischen Anforderungen des positivistischen Fallibilismus, sondern eine inakzeptable Zugangsbeschränkung zum ,wissenschaftlichen Markt“ dieser sich verweigernden Ansätze, die mittlerweile vielfach dokumentiert ist (vgl. u.a. AFEP 2009; Corsi et al. 2010; Heise und Thieme 2016) - und niemand kann sagen, er hätte es nicht gewusst (oder zumindest wissen können). Es geht also in keinem Fall darum, vom Aussterben bedrohte Paradigmen unter Artenschutz stellen zu wollen, sondern heterodoxe Paradigmen können selbstbewusst auf den Pluralismusimperativ einer Sozialwissenschaft pochen (vgl. Heise 2017).

Leider werden die eigentlichen inhaltlichen Diskussionen, um die es in der Wissenschaft vordringlich gehen sollte, allzu häufig von diesen wissenschaftstheoretischen und -sozio-logischen Betrachtungen an den Rand gedrängt. Dies gilt leider auch für den Kommentar Johannes Beckers, wo nur wenige inhaltliche Diskussionsfragen aufgeworfen werden. Der Vollständigkeit halber sollen diese dennoch beantwortet werden: Dass Wechselkursfixierungen Bewertungsvolatilitäten reduzieren - in diesem Falle des Außenwertes von Waren und Dienstleistungen - und damit Unsicherheiten reduzieren helfen, ist keine „eigentümliche Behauptung“, sondern unabweisbarer Fakt. Dem steht auch nicht entgegen, dass mit der vollständigen Wechselkursfixierung z.B. in einer Währungsunion Anpassungskosten an anderer Stelle entstehen. Und selbst der Anstieg der Risikoprämien auf manche südländische Staatsanleihe in der Eurozone - worauf Becker mit den „katastrophalen Erfahrungen der Südländer in der Eurokrise" vermutlich anspielt - ist eben nicht ursächlich auf die Wechselkursfixierung zurückzuführen, sondern spiegelt im Gegenteil die Unsicherheit über den Bestand der Währungsunion - also den Fortbestand der Wechselkursfixierung - wider (vgl. Pusch 2016). Und schließlich möchte ich darauf verweisen, dass im Rahmen des vorgestellten Postkeynesianismus die Unabhängigkeit von Zentralbanken besser begründet werden kann als in DSGMen, die eine monetäre Neutralität und die Formbarkeit rationaler Erwartungen (z.B. über das geldpolitische Verhalten abhängiger Zentralbanken) postulieren (Heise 2015). Eine auch nur implizite Forderung nach Aufgabe der Unabhängigkeit der Zentralbank kann also zumindest dem hier vorgestellten Postkeynesianismus nicht unterstellt werden - gleichwohl darf „Unabhängigkeit“ nicht mit „Kooperationsverweigerung“ gleichgesetzt werden. Dies würde die Erkenntnisse der „Policy Games“-Literatur (z.B. Nordhaus 1994; Iversen et al. 2000; Heise 2008) negieren. 


\section{Literatur}

Association Francaise d'Economie Politique (AFEP) (2009). Evolution of the economics professors' recruitment since 2000 in France. The End of Pluralism. http://www.assoeconomiepolitique.org/wpcontent/uploads/FAPE-State-of-Pluralism-in-France-Final-Version.pdf. Zugegriffen: 13.02.2019

Corsi, M., D'Ippoliti, C., \& Lucisi, F. (2010). Pluralism at risk? Heterodox economic approaches and the evaluation of economicresearch in Italy. The American Journal of Economics and Sociology, 69(5), 1495-1529.

Davidson, P. (2007). John Maynard Keynes. Houndsmill: Palgrave MacMillan.

Heise, A. (2008). Economic governance and employment. Policy, Polity and Politics of Economic Rise and Decline, Münster: Lit Verlag.

Heise, A. (2015). Eintrag 'Central bank independence. In L.-P. Rochon \& S. Rossi (Hrsg.), The encyclopedia of central banking (S. 85-87). Cheltenham: Edward Elgar.

Heise, A. (2016). Why has economics turned out this way? A socio-economic note on the explanation of monism in economics. Journal of Philosophical Economics, 10(1), 81-101.

Heise, A. (2017). Defining economic pluralism: ethical norm or scientific imperative. International Journal for Pluralism and Economic Education, 8(1), 18-41.

Heise, A., \& Thieme, S. (2016). The short rise and long fall of heterodox economics. Explorations in a scientific field of power and struggle. Journal of Economic Issues, 50(4), 1105-1130.

Iversen, T., Pontussen, J., \& Soskice, D. (Hrsg.). (2000). Unions, employers, and central banks. Macroeconomic coordination and institutional change in social market economies. Cambridge: University Press.

Kapeller, J. (2010a). Some critical notes on citation metrics and heterodox economics. Review of Radical Political Economics, 42(3), 330-337.

Kapeller, J. (2010b). Citation metrics: serious drawbacks, perverse incentives and strategic options for heterodox economics. American Journal of Economics and Sociology, 69(5), 1376-1408.

Nordhaus, W.D. (1994). Policy games. Coordination and independence in monetary and fiscal policies. Brookings Papers on Economic Activity, (2), 139-216.

Pusch, T. (2016). The role of uncertainty in the euro crisis - an application of liquidity preference theory. International Review of Applied Economics, 31(4), 1-22.

Schumpeter, J. A. (1954). History of economic analysis. London: Allen \& Unwin. 